\title{
Seed Health and Quality Test of Three Rice Varieties for the Detection of Fungi Associated with Seed Sample
}

\author{
Mansur Ahmed $^{1}$, Mehbub Hossain ${ }^{1}$, Kamrul Hassan ${ }^{2, *}$,Chandra Kanta Dash ${ }^{2}$ \\ ${ }^{1}$ Department of Plant Pathology, Bangladesh Agricultural University, Mymensingh-2202 \\ ${ }^{2}$ Department of Entomology, Sylhet Agricultural University, Sylhet-3100 \\ *Corresponding Author: shishir244@gmail.com
}

Copyright (C) 2013 Horizon Research Publishing All rights reserved.

\begin{abstract}
The experiment was carried out at the Seed Pathology Centre (SPC) and MS Laboratory of the Department of Plant Pathology, Bangladesh Agricultural University (BAU), Mymensingh during the period of August, 2008 to October, 2009 to detect the fungi associate with the seed samples and to record the germination of seed samples of Parshuram upazila of Feni district. Three rice varieties are collected for the studies were BR6, Pajam and Joya (Local) from Parshuram upazila of Feni district to determine the seed health and quality. For determining the seed health and quality, Dry inspection and blotter tests were performed. Apparently healthy seeds (61.50-78.00\%), spotted seeds (6.15-12.90\%), discoloured seeds (4.80-14.25\%), deformed seeds $(2.00-7.25 \%)$, varietal mixtures $(2.20-9.80 \%)$ and chaffy grains $(0.95-6.50 \%)$ were found among the three rice varieties. The germination of rice seeds of the variety BR6 was $54.67 \%$, while the varieties joya and Pajam showed $58.00 \%$ germination respectively. Nine seed-borne fungi were detected from these seed samples. The identified fungi were Fusarium oxysporum, F. moniliforme, Bipolaris oryzae, Alternaria padwickii, Curvularia lunata, Aspergillus flavus, Aspergillus niger, Penicillium sp. and Nigrospora oryzae. Seeds of rice variety Joya and Pajam carried the lower infection of all the seed-borne fungi than the variety BR6.
\end{abstract}

Keywords Seed Health and Quality, Dry Inspection, Identification of Fungi

\section{Introduction}

Rice (Oryza sativa) is the staple food of Bangladeshi people and it constituted about $90 \%$ of the total food grain production [1]. It covers about 75 percent of the total cultivable land in Bangladesh [2]. The average world yield of rice is 3.84 tons/ha [2], but the average yield of rice in Bangladesh is only $2.52 \mathrm{t} \mathrm{ha-}^{-}{ }^{1}[3]$. So the average per hectare production of rice in Bangladesh is extremely low as compared to other rice growing countries of the world. In Bangladesh, out of $16 \%$ annual crop losses due to plant diseases, at least $10 \%$ loss is incurred due to seed-borne diseases [4]. In our country farmer's saved seeds are the main source of seeds for rice cultivation, but the farmers do not maintain the seed health properly that resulting heavy infestation by the fungi, particularly during hot and humid seasons and cause deterioration of quality and viability of seeds. These seeds results in reduced germination rate and transmit pathogens from seed to seed bed and ultimately cause field diseases. Reduction in yield and quality loss of grains of rice ranges from a trace to total crop loss depending on the inoculum density, pathogen aggressiveness, environmental conditions, cultivar susceptibility and interaction with other cultural parameters [5].Consedering above situation the present study was under taken to detect the fungi associate with the seed samples of Parshuram upazila of Feni district and record the germination of seed samples.

\section{Materials and Method}

The experiment was carried out at the Seed Pathology Centre (SPC) and MS Laboratory of the Department of Plant Pathology, Bangladesh Agricultural University (BAU), Mymensingh.Three rice varieties selected for the study were BR6, Pajam and Joya .The experiment was conducted following Completely Randomized Design (CRD) with three replications. A total of 36 rice seed samples were collected from farmers of Porshuram Upazila of Feni District. Twelve seed samples were collected from different part of the upazila for each rice variety. The samples were enclosed in paper bags with proper labeling, brought to the SPC laboratory and kept in the refrigerator at $5 \pm 1{ }^{\circ} \mathrm{C}$ until used for subsequent studies.

\subsection{Dry Inspection of Seed Samples}

400 seeds from each sample were visually inspected and graded into six categories. The categories were: (1) Apparently healthy seeds, (2) Spotted seeds, (3) Discoloured seeds, (4) Deformed seeds, (5) Varietal mixture and (6) Chaffy grain. Separation of different categories of seeds was 
done with unaided eyes. The different seed categories were expressed in percentage.

\subsection{Detection of seed-borne fungi}

All the seed samples were assayed for the presence of fungal pathogens by the Standard Blotter Method following the rules of International Seed Testing Association [6].

\subsection{Plating of seeds}

To detect the seed-borne fungal pathogens associated with the seeds in seed samples the Blotter method was used by following international rules for seed testing (ISTA, 1976). In this method, three layers of blotting papers (Whatman No. 1) were soaked in sterile water and placed at the bottom of 9 $\mathrm{cm}$ diameter petridishes. Four hundred seeds from each sample were taken randomly and then placed on the moist filter paper at the rate of 25 seeds per plate. The petridishes with seeds were then incubated at $22 \pm 2{ }^{\circ} \mathrm{C}$ under $12 / 12$ alternating, cycles of Near Ultra-Violet (NUV) light and darkness in the incubation room of the SPC, BAU, Mymensingh for seven days. Time to time watering was done to keep the filter paper moist. After incubation, the seeds were examined under the stereo-microscope for the presence of seed-borne fungi. Data were expressed in percentage. The rice variety of the particular sample showing highest incidence of seed-borne infection was selected for seed treatment.

\subsection{Identification of fungi}

Each individual seed was observed under stereo-microscope at X16 and X25 magnification in order to identify the seed-borne fungi. Most of the associated fungi were detected by observing their growth characters on the incubated seeds following the keys outlined by [7] [8] [9] [10]. Temporary slides were also prepared and observed under compound microscope for proper identification. The fungi were identified to species level, wherever possible, following the keys of [11] [12] [13] [14]. The results were presented as percent incidence for individual pathogen.

\subsection{Statistical Analysis}

The data collected from the experiment were analyzed for test of significance and compared the treatment means following Completely Randomized Design (CRD) by using Duncan's Multiple Range Test (DMRT) at 5\% level of probability.

\section{Result and Discussion}

\subsection{Dry inspections}

Dry inspections of 400 seeds of each of 12 samples for three varieties were done and they were categorized in six components, they were apparently healthy seed, spotted seed, discoloured seed, deformed seed, varietal mixture and chaffy grain. In case of variety Pajam, the samples contained 62.16 to $78.00 \%$ apparently healthy seeds, 6.15 to $9.45 \%$ spotted seeds, 4.80 to $9.25 \%$ discoloured seeds, and 2.85 to $7.25 \%$ deformed seeds and 2.45 to $8.70 \%$ varietal mixtures and 1.05 to $5.14 \%$ chaffy grains (Figure 1 ). These results varied from variety to variety and location to location. In case of variety BR6, apparently healthy seeds ranged from 65.70 to $70.50 \%$. The highest $70.50 \%$ apparently healthy seeds were recorded in S8 sample. It has been recorded that the collected seed samples contained 8.58 to $11.90 \%$ spotted seeds, 5.60 to $10.68 \%$ discoloured seeds, and 2.00 to $6.85 \%$ deformed seeds, 2.20 to $9.80 \%$ varietal mixtures and 1.79 to $4.45 \%$ chaffy grains (Figure 2). Rice seeds available in different parts of Bangladesh contain deformed and discoloured seeds as found in the present investigation was supported by the reports of [13] who recorded 14.43 to $24.44 \%$ discolored and 8.46 to $15.50 \%$ deformed seeds of rice in Rajshahi, Rangpur and Bogra districts. This variation may be due to the variations of farmers' knowledge regarding the seed production \& processing. [14] and [15] who reported 4.35 to $79.82 \%$ discolored and 12.30 to $14.14 \%$ spotted seeds in rice seeds. During the present investigation nine species of fungi were identified on the seeds of three varieties. The identified fungi were Fusarium oxysporum, F. moniliforme, Bipolaris oryzae, Aspergillus flavus, Alternaria padwickii, Curvularia lunata, Aspergillus niger, Penicillium sp. and Nigrospora oryzae. Highest seed infection, was recorded for Bipolaris oryzae followed by that of Alternaria padwickii, Curvularia lunata, Fusarium oxysporum, F. moniliforme, Aspergillus flavus, Penicillium sp., Nigrospora oryzae and the lowest percentage of Aspergillus niger.Percent seed-borne infection in rice variety BR6 was higher and it yielded most of the fungi at a higher percentage than Joya and Pajam (Table 4). The incidence of Alternaria padwickii was $12.67 \%$ in BR6, $10.00 \%$ in seeds Joya and $6.667 \%$ in Pajam. The incidence of Alternaria padwickii was significantly different from Joya and Pajam. The incidence of Bipolaris oryzae in the seeds of three rice varieties BR6, Joya and Pajam were $13.00 \%$, $12.33 \%$ and $11.333 \%$, respectively. There is no statistically significant difference among three varieties in case Bipolaris oryzae. Incidence of Curvularia lunata in seeds of BR6 $(11.00 \%)$ was significantly different from Joya $(5.333 \%)$ and Pajam (4.667\%). Percentage of Aspergillus niger was significantly higher in seeds of Pajam $(3.00 \%)$ and BR6 $(2.667 \%)$ than Joya $(0.00 \%)$. Seeds of Pajam showed significant difference in the incidence of Aspergillus flavus than Joya and BR6 and it was $8.667 \%, 5.333 \%$ and $4.667 \%$ in Pajam, BR6 and Joya, respectively. Incidence of Fusarium oxysporum in the seeds of three rice varieties Joya, BR6 and Pajam was 9.333\%, $7.000 \%$ and $6.333 \%$, respectively. The difference was statistically significant. Incidence of Fusarium moniliforme in the seeds of three rice varieties Joya, BR6 and Pajam was 6.333\%, 6.333\% and $4.667 \%$, respectively. Incidence of Nigrospora oryzae in the 
seeds of three rice varieties Joya, BR6 and Pajam was 4.00\%, $6.667 \%$ and $4.00 \%$, respectively. Incidence of Penecillium $s p$ in the seeds of three rice varieties Joya, BR6 and Pajam was $5.00 \%, 4.667 \%$ and $4.667 \%$, respectively. There was no significant difference in case of Fusarium moniliforme, Nigrospora oryzae and Penicillium sp.

\section{Conclusion}

The results of this study indicate that there is a significance variation for seed health and quality among the three varieties of rice. The present findings will help the researchers for further investigations and ultimately our farmers to use healthy and quality seed.

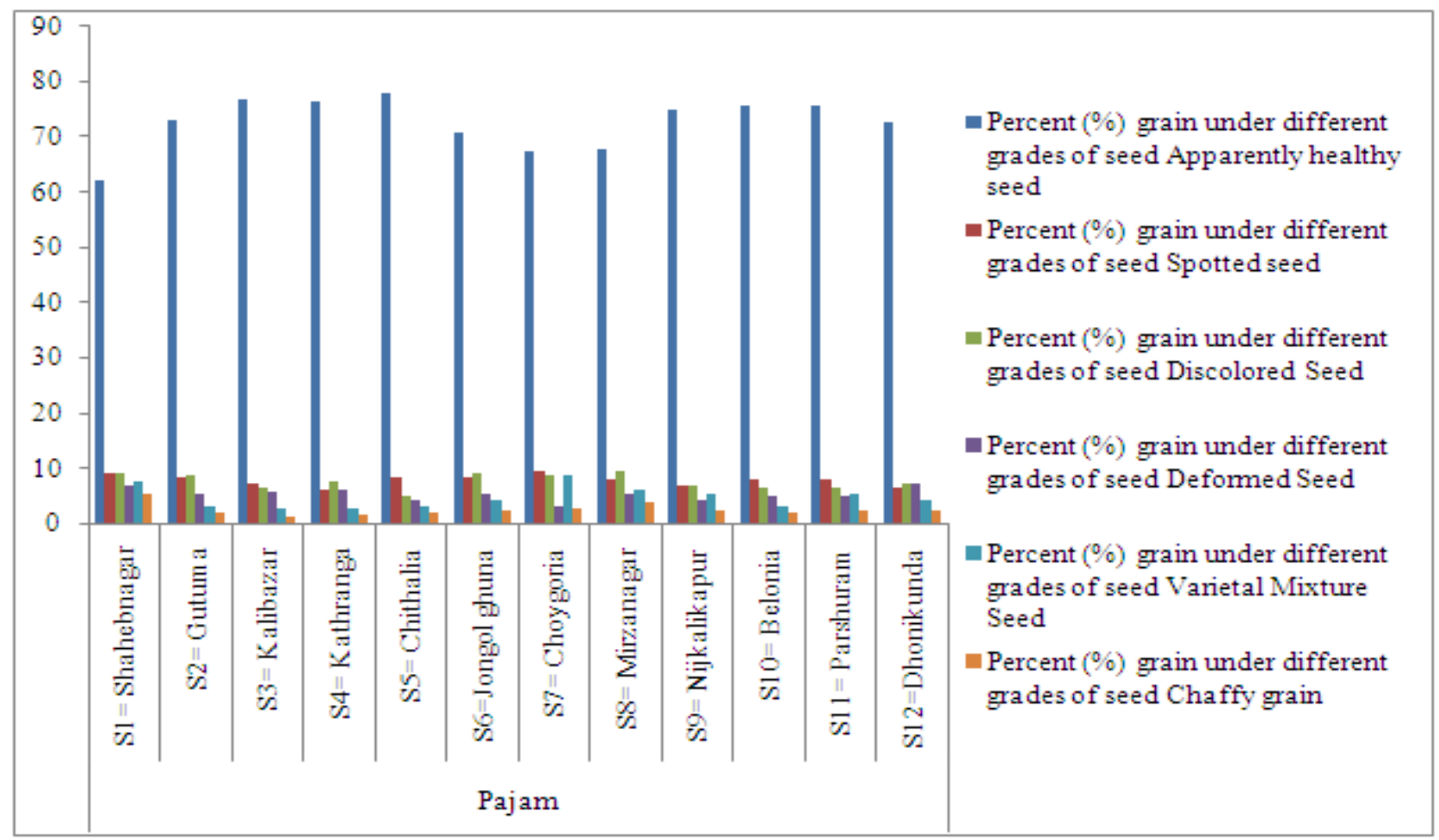

Figure 1. Graphical representation of rice seed sample (Variety: pajam) after dry inspection

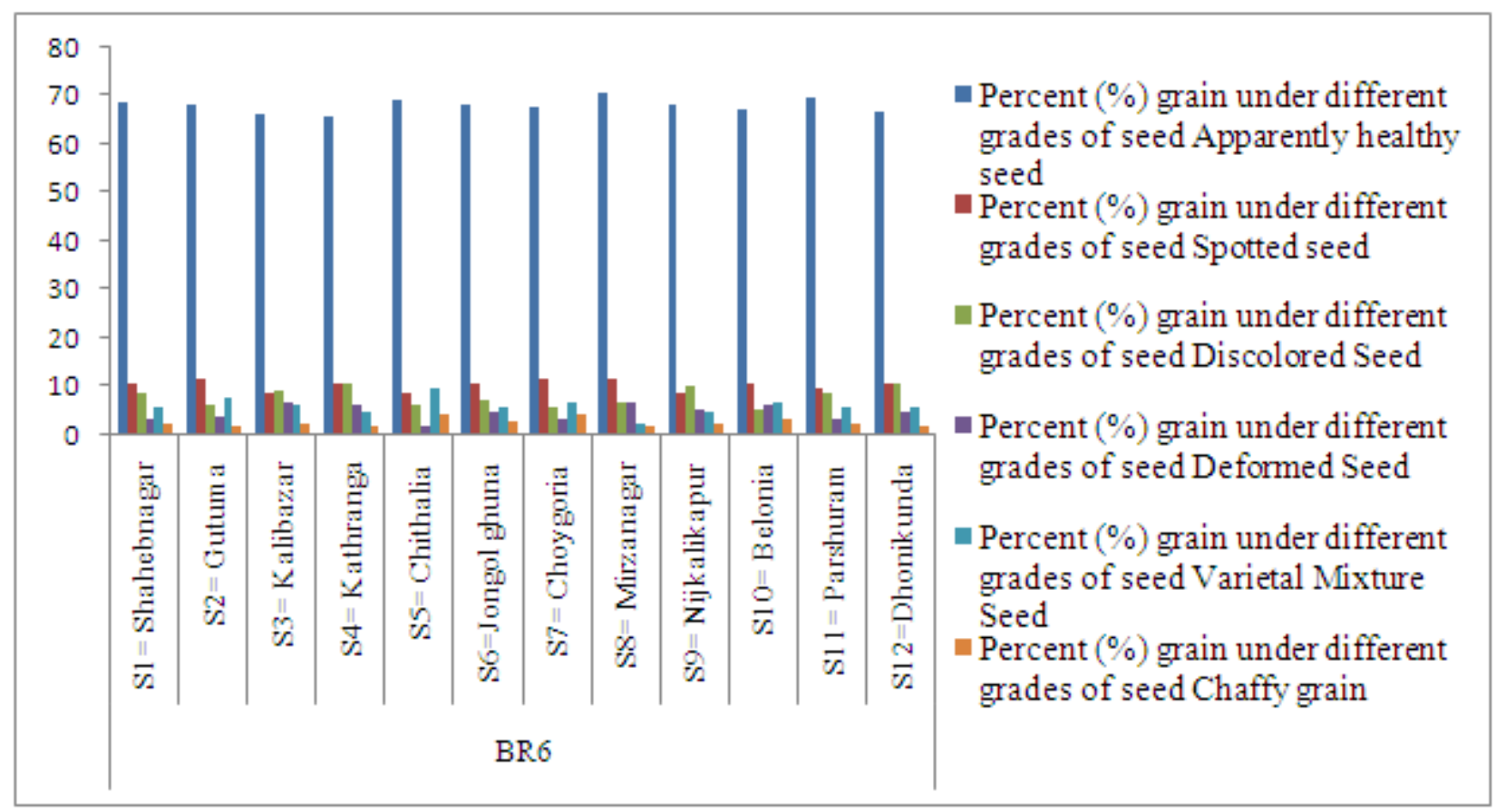

Figure 2. Graphical representation of rice seed sample (Variety: BR6) after dry inspection 


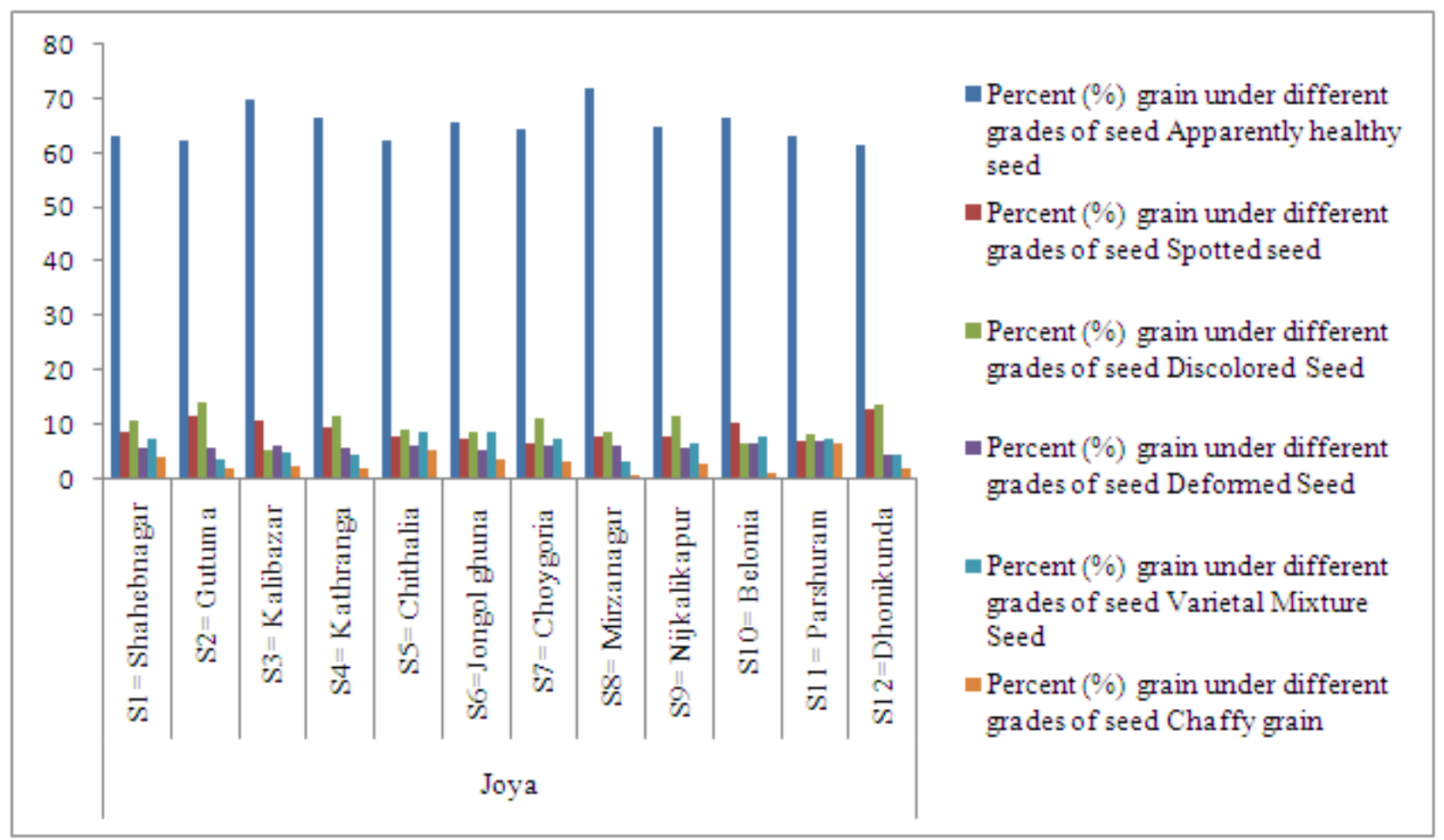

Figure 3. Graphical representation of rice seed sample (Variety: Joya) after dry inspection

Table 1. The germination percentage and prevalence of fungi associated with seeds of Variety Pajam

\begin{tabular}{|c|c|c|c|c|c|c|c|c|c|c|}
\hline Sample & $\begin{array}{l}\text { Germinat } \\
\text { ion }\end{array}$ & $\begin{array}{l}\text { Alternari } \\
\quad a\end{array}$ & $\begin{array}{c}\text { Aspergill } \\
\text { us }\end{array}$ & $\begin{array}{c}\text { Bipolaris } \\
\text { oryzae }\end{array}$ & $\begin{array}{l}\text { Curvulari } \\
\text { a lunata }\end{array}$ & $\begin{array}{l}\text { Fusarium } \\
\text { monilifor }\end{array}$ & $\begin{array}{l}\text { Fusarium } \\
\text { oxysporum }\end{array}$ & $\begin{array}{c}\text { Nigrospor } \\
a\end{array}$ & $\begin{array}{c}\text { Aspergill } \\
\text { us }\end{array}$ & $\begin{array}{l}\text { Penicilliu } \\
m \text { sp. }\end{array}$ \\
\hline $\begin{array}{c}\mathrm{S}_{1}= \\
\text { Shahebnagar }\end{array}$ & $72.33 \mathrm{~g}$ & $3.667 \mathrm{de}$ & $1.667 \mathrm{~b}$ & $8.333 \mathrm{bc}$ & $3.333 \mathrm{abc}$ & $2.333 \mathrm{cde}$ & $2.000 \mathrm{de}$ & $1.667 \mathrm{~b}$ & $4.000 \mathrm{~b}$ & $2.333 \mathrm{~b}$ \\
\hline $\mathrm{S}_{2}=$ Gutuma & $78.33 \mathrm{bcd}$ & $4.667 \mathrm{bcd}$ & $1.667 \mathrm{~b}$ & $8.667 \mathrm{~b}$ & $2.667 \mathrm{c}$ & $3.667 \mathrm{ab}$ & $4.000 \mathrm{~b}$ & $0.000 \mathrm{c}$ & $3.667 \mathrm{bc}$ & $2.000 \mathrm{bc}$ \\
\hline $\begin{array}{c}\mathrm{S}_{3}= \\
\text { Kalibazar }\end{array}$ & $67.33 \mathrm{~h}$ & $4.000 \mathrm{cde}$ & $0.000 \mathrm{~d}$ & $8.667 \mathrm{~b}$ & $4.333 \mathrm{ab}$ & $3.333 \mathrm{bc}$ & $2.333 \mathrm{cde}$ & $0.333 \mathrm{c}$ & $2.333 \mathrm{~d}$ & $0.667 \mathrm{de}$ \\
\hline $\begin{array}{c}\mathrm{S}_{4}= \\
\text { Kathranga }\end{array}$ & $75.67 \mathrm{f}$ & $\begin{array}{l}4.333 \\
\text { bcde }\end{array}$ & $1.000 \mathrm{c}$ & $8.667 \mathrm{~b}$ & $2.667 \mathrm{c}$ & $1.667 \mathrm{ef}$ & $2.000 \mathrm{de}$ & $0.000 \mathrm{c}$ & $2.667 \mathrm{~cd}$ & $0.333 \mathrm{e}$ \\
\hline $\begin{array}{c}\mathrm{S}_{5}= \\
\text { Chithalia }\end{array}$ & 77.67 cde & $3.333 \mathrm{de}$ & $0.000 \mathrm{~d}$ & $8.667 \mathrm{~b}$ & $3.000 \mathrm{bc}$ & $2.000 \mathrm{de}$ & $1.333 \mathrm{e}$ & $0.333 \mathrm{c}$ & $3.667 \mathrm{bc}$ & $1.667 \mathrm{bcd}$ \\
\hline $\begin{array}{c}\mathrm{S}_{6}=\text { Jongol } \\
\text { ghuna }\end{array}$ & $79.33 \mathrm{bc}$ & $6.000 \mathrm{ab}$ & $0.000 \mathrm{~d}$ & $8.333 \mathrm{bc}$ & $4.667 \mathrm{a}$ & $\begin{array}{l}2.667 \\
\text { bcde }\end{array}$ & $3.667 \mathrm{~b}$ & $0.000 \mathrm{c}$ & $2.667 \mathrm{~cd}$ & $1.333 \mathrm{cde}$ \\
\hline $\begin{array}{c}\mathrm{S}_{7}= \\
\text { Choygoria }\end{array}$ & $81.67 \mathrm{a}$ & $2.667 \mathrm{e}$ & $0.000 \mathrm{~d}$ & $7.333 \mathrm{bc}$ & $3.333 \mathrm{abc}$ & $1.667 \mathrm{ef}$ & $3.000 \mathrm{bcd}$ & $0.000 \mathrm{c}$ & $2.667 \mathrm{~cd}$ & $1.333 \mathrm{cde}$ \\
\hline $\begin{array}{c}\mathrm{S}_{8}= \\
\text { Mirzanagar }\end{array}$ & $58.00 \mathrm{i}$ & $6.667 \mathrm{a}$ & $3.000 \mathrm{a}$ & $11.333 \mathrm{a}$ & $4.667 \mathrm{a}$ & $4.667 \mathrm{a}$ & $6.333 \mathrm{a}$ & $4.000 \mathrm{a}$ & $8.667 \mathrm{a}$ & $4.667 \mathrm{a}$ \\
\hline $\begin{array}{c}\mathrm{S}_{9}= \\
\text { Nijkalikapur }\end{array}$ & $71.33 \mathrm{~g}$ & $5.557 \mathrm{abc}$ & $0.000 \mathrm{~d}$ & $8.667 \mathrm{~b}$ & $2.667 \mathrm{c}$ & $2.333 \mathrm{cde}$ & $2.000 \mathrm{de}$ & $0.000 \mathrm{c}$ & $2.667 \mathrm{~cd}$ & $2.000 \mathrm{bc}$ \\
\hline $\mathrm{S}_{10}=$ Belonia & $76.33 \mathrm{ef}$ & $4.667 \mathrm{bcd}$ & $0.000 \mathrm{~d}$ & $7.000 \mathrm{bc}$ & $3.333 \mathrm{abc}$ & $3.000 \mathrm{bcd}$ & $3.667 \mathrm{~b}$ & $0.667 \mathrm{c}$ & $4.667 \mathrm{~b}$ & $0.667 \mathrm{e}$ \\
\hline $\begin{array}{c}\text { s11 }= \\
\text { Parshuram }\end{array}$ & $77.00 \mathrm{def}$ & $4.000 \mathrm{cde}$ & $1.333 \mathrm{bc}$ & $6.667 \mathrm{c}$ & $3.333 \mathrm{abc}$ & $0.667 \mathrm{f}$ & $1.667 \mathrm{e}$ & $0.000 \mathrm{c}$ & $2.000 \mathrm{~d}$ & $1.333 \mathrm{cde}$ \\
\hline $\begin{array}{c}\mathrm{S}_{12}=\text { Dhoniku } \\
\text { nda }\end{array}$ & $80.00 \mathrm{ab}$ & $3.333 \mathrm{de}$ & $0.000 \mathrm{~d}$ & $4.667 \mathrm{~d}$ & $4.000 \mathrm{abc}$ & $2.333 \mathrm{cde}$ & $3.333 \mathrm{bc}$ & $0.000 \mathrm{c}$ & $2.667 \mathrm{~cd}$ & $1.333 \mathrm{cde}$ \\
\hline
\end{tabular}

Data presents the mean of three replications

Three hundred seeds were tested for each sample

Figure with common letters did not differ significantly at $5 \%$ level by DMRT 
Table 2. The germination percentage and prevalence of fungi associated with seeds of Variety BR6

\begin{tabular}{|c|c|c|c|c|c|c|c|c|c|c|}
\hline Sample & $\begin{array}{l}\text { Germinati } \\
\text { on }\end{array}$ & $\begin{array}{l}\text { Alterna } \\
\text { ria } \\
\text { padwic } \\
\text { kii }\end{array}$ & $\begin{array}{c}\text { Aspergill } \\
\text { us } \\
\text { niger }\end{array}$ & $\begin{array}{c}\text { Bipolari } \\
s \\
\text { oryzae }\end{array}$ & $\begin{array}{c}\text { Curvulari } \\
\text { a } \\
\text { lunata }\end{array}$ & $\begin{array}{c}\text { Fusarium } \\
\text { monilifor } \\
\text { me }\end{array}$ & $\begin{array}{c}\text { Fusarium } \\
\text { oxysporu } \\
m\end{array}$ & $\begin{array}{c}\text { Nigrospor } \\
a \\
\text { oryzae }\end{array}$ & $\begin{array}{c}\text { Aspergill } \\
\text { us } \\
\text { flavus }\end{array}$ & $\begin{array}{c}\text { Penicillium } \\
s p .\end{array}$ \\
\hline $\begin{array}{c}\mathrm{S}_{1}= \\
\text { Shahebnagar }\end{array}$ & $77.73 \mathrm{c}$ & $7.333 \mathrm{c}$ & $1.667 \mathrm{~b}$ & $\begin{array}{c}12.00 \\
\mathrm{ab}\end{array}$ & $3.000 \mathrm{~d}$ & $4.667 \mathrm{abcd}$ & $3.000 \mathrm{bcd}$ & $0.000 \mathrm{c}$ & $3.333 \mathrm{bc}$ & $1.333 \mathrm{bc}$ \\
\hline $\mathrm{S}_{2}=$ Gutuma & $72.67 \mathrm{~d}$ & $\begin{array}{c}7.667 \\
\mathrm{bc}\end{array}$ & $0.000 \mathrm{~d}$ & $\begin{array}{l}7.667 \\
\text { cde }\end{array}$ & $7.000 \mathrm{~b}$ & $5.333 \mathrm{ab}$ & $4.6667 \mathrm{~b}$ & $0.000 \mathrm{c}$ & $4.333 \mathrm{ab}$ & $0.667 \mathrm{c}$ \\
\hline $\mathrm{S}_{3}=$ Kalibazar & $54.67 \mathrm{f}$ & $12.67 \mathrm{a}$ & $2.667 \mathrm{a}$ & $13.00 \mathrm{a}$ & $11.00 \mathrm{a}$ & $6.333 \mathrm{a}$ & $7.000 \mathrm{a}$ & $3.667 \mathrm{a}$ & $5.333 \mathrm{a}$ & $4.667 \mathrm{a}$ \\
\hline $\mathrm{S}_{4}=$ Kathranga & $77.00 \mathrm{c}$ & $7.333 \mathrm{c}$ & $0.000 \mathrm{~d}$ & $\begin{array}{l}7.333 \\
\text { cde }\end{array}$ & $5.000 \mathrm{bcd}$ & $3.000 \mathrm{de}$ & $2.333 \mathrm{~cd}$ & $0.000 \mathrm{c}$ & $3.000 \mathrm{bc}$ & $1.000 \mathrm{c}$ \\
\hline $\mathrm{S}_{5}=$ Chithalia & $81.33 \mathrm{~b}$ & $7.333 \mathrm{c}$ & $0.000 \mathrm{~d}$ & $\begin{array}{c}9.333 \\
\text { bcd }\end{array}$ & $5.000 \mathrm{bcd}$ & $0.000 \mathrm{f}$ & $3.333 \mathrm{bcd}$ & $0.000 \mathrm{c}$ & $4.000 \mathrm{ab}$ & $1.667 \mathrm{bc}$ \\
\hline $\begin{array}{c}\mathrm{S}_{6}=\text { Jongol } \\
\text { ghuna }\end{array}$ & $69.33 \mathrm{e}$ & $9.667 \mathrm{~b}$ & $0.000 \mathrm{~d}$ & $\begin{array}{c}12.33 \\
\mathrm{ab}\end{array}$ & $4.000 \mathrm{~cd}$ & $2.333 \mathrm{e}$ & $2.000 \mathrm{~cd}$ & $1.333 \mathrm{~b}$ & $3.000 \mathrm{bc}$ & $1.333 \mathrm{bc}$ \\
\hline $\mathrm{S}_{7}=$ Choygoria & $70.33 \mathrm{e}$ & $\begin{array}{c}8.333 \\
\mathrm{bc}\end{array}$ & $0.000 \mathrm{~d}$ & $\begin{array}{c}11.00 \\
\mathrm{ab}\end{array}$ & $5.333 \mathrm{bcd}$ & $4.667 \mathrm{abcd}$ & $3.000 \mathrm{bcd}$ & $0.000 \mathrm{c}$ & $4.667 \mathrm{ab}$ & $2.000 \mathrm{bc}$ \\
\hline $\mathrm{S}_{8}=$ Mirzanagar & $81.00 \mathrm{~b}$ & $\begin{array}{c}8.333 \\
b c\end{array}$ & $0.667 \mathrm{c}$ & $\begin{array}{c}11.33 \\
\mathrm{ab}\end{array}$ & $5.333 \mathrm{bcd}$ & 3.667 bcde & $4.333 \mathrm{~b}$ & $0.000 \mathrm{c}$ & $3.000 \mathrm{bc}$ & $2.000 \mathrm{bc}$ \\
\hline $\mathrm{S}_{9}=$ Nijkalikapur & $81.67 \mathrm{~b}$ & $6.667 \mathrm{c}$ & $0.000 \mathrm{~d}$ & $\begin{array}{c}10.33 \\
\mathrm{abc}\end{array}$ & $4.667 \mathrm{~cd}$ & $4.333 \mathrm{bcd}$ & $3.667 \mathrm{bc}$ & $0.000 \mathrm{c}$ & $4.667 \mathrm{ab}$ & $1.333 \mathrm{bc}$ \\
\hline $\mathrm{S}_{10}=$ Belonia & $85.00 \mathrm{a}$ & $7.333 \mathrm{c}$ & $0.000 \mathrm{~d}$ & $\begin{array}{c}6.333 \\
\text { de }\end{array}$ & $3.000 \mathrm{~d}$ & $2.333 \mathrm{e}$ & $3.000 \mathrm{bcd}$ & $1.000 \mathrm{~b}$ & $3.333 \mathrm{bc}$ & $2.667 \mathrm{bc}$ \\
\hline $\mathrm{s} 11=$ Parshuram & $76.67 \mathrm{c}$ & $6.667 \mathrm{c}$ & $0.000 \mathrm{~d}$ & $\begin{array}{c}7.000 \\
\text { de }\end{array}$ & $4.333 \mathrm{~cd}$ & $3.333 \mathrm{cde}$ & $1.667 \mathrm{~d}$ & $0.000 \mathrm{c}$ & $2.000 \mathrm{c}$ & $1.333 \mathrm{c}$ \\
\hline $\mathrm{S}_{12}=$ Dhonikunda & $74.33 \mathrm{~d}$ & $7.000 \mathrm{c}$ & $0.000 \mathrm{~d}$ & $6.000 \mathrm{e}$ & $6.333 \mathrm{bc}$ & $5.000 \mathrm{abc}$ & $4.667 \mathrm{~b}$ & $0.000 \mathrm{c}$ & $3.667 \mathrm{abc}$ & $1.333 \mathrm{c}$ \\
\hline
\end{tabular}

Data presents the mean of three replications

Three hundred seeds were tested for each sample

Figure with common letters did not differ significantly at $5 \%$ level by DMRT

Table 3. The germination percentage and prevalence of fungi associated with seeds of Variety Joya

\begin{tabular}{|c|c|c|c|c|c|c|c|c|c|c|}
\hline Sample & $\begin{array}{l}\text { Germinat } \\
\text { in }\end{array}$ & $\begin{array}{c}\text { Alternaria } \\
\text { padwickii }\end{array}$ & $\begin{array}{c}\text { Aspergill } \\
\text { us } \\
\text { niger }\end{array}$ & $\begin{array}{c}\text { Bipolaris } \\
\text { oryzae }\end{array}$ & $\begin{array}{c}\text { Curvular } \\
\text { ia } \\
\text { lunata }\end{array}$ & $\begin{array}{c}\text { Fusarium } \\
\text { monilifor } \\
\text { me }\end{array}$ & $\begin{array}{l}\text { Fusarium } \\
\text { oxysporum }\end{array}$ & $\begin{array}{c}\text { Nigrospora } \\
\text { oryzae }\end{array}$ & $\begin{array}{c}\text { Aspergill } \\
\text { us } \\
\text { flavus }\end{array}$ & $\begin{array}{c}\text { Penicillium } \\
\text { sp. }\end{array}$ \\
\hline $\begin{array}{c}\mathrm{S}_{1}= \\
\text { Shahebnagar }\end{array}$ & $\begin{array}{c}75.67 \\
b c\end{array}$ & $3.667 \mathrm{~d}$ & $0.000 \mathrm{a}$ & $11.33 \mathrm{ab}$ & $\begin{array}{c}3.667 \\
\text { bcd }\end{array}$ & $3.333 \mathrm{bc}$ & $2.333 \mathrm{bc}$ & $0.000 \mathrm{c}$ & $2.667 \mathrm{abc}$ & $3.333 \mathrm{~b}$ \\
\hline $\mathrm{S}_{2}=$ Gutuma & 69.63 ef & $6.667 \mathrm{bc}$ & $0.000 \mathrm{a}$ & $\begin{array}{l}7.667 \\
\text { bcde }\end{array}$ & $3.000 \mathrm{~cd}$ & $3.667 \mathrm{bc}$ & $3.000 \mathrm{bc}$ & $0.000 \mathrm{c}$ & $2.667 \mathrm{abc}$ & $1.667 \mathrm{~cd}$ \\
\hline $\begin{array}{c}\mathrm{S}_{3}= \\
\text { Kalibazar }\end{array}$ & $68.67 \mathrm{f}$ & $4.667 \mathrm{~cd}$ & $0.000 \mathrm{a}$ & $\begin{array}{l}8.000 \\
\text { bcde }\end{array}$ & $2.667 \mathrm{~cd}$ & $2.333 \mathrm{bc}$ & $2.000 \mathrm{c}$ & $0.333 \mathrm{c}$ & $3.667 \mathrm{ab}$ & $3.000 \mathrm{bc}$ \\
\hline $\begin{array}{c}\mathrm{S}_{4}= \\
\text { Kathranga }\end{array}$ & $75.33 \mathrm{~cd}$ & $4.333 \mathrm{~cd}$ & $0.000 \mathrm{a}$ & $\begin{array}{l}9.333 \\
\text { abcd }\end{array}$ & $\begin{array}{l}4.000 \\
\text { abcd }\end{array}$ & $2.667 \mathrm{bc}$ & $2.000 \mathrm{c}$ & $0.333 \mathrm{c}$ & $1.667 \mathrm{bc}$ & $1.333 \mathrm{~d}$ \\
\hline $\begin{array}{c}\mathrm{S}_{5}= \\
\text { Chithalia }\end{array}$ & $78.67 \mathrm{ab}$ & $4.667 \mathrm{~cd}$ & $0.000 \mathrm{a}$ & $10.00 \mathrm{abc}$ & $\begin{array}{c}3.667 \\
\text { bcd }\end{array}$ & $4.333 \mathrm{~b}$ & $2.667 \mathrm{bc}$ & $0.667 \mathrm{c}$ & $2.667 \mathrm{abc}$ & $3.000 \mathrm{bc}$ \\
\hline $\begin{array}{c}\mathrm{S}_{6}=\text { Jongol } \\
\text { ghuna }\end{array}$ & $58.00 \mathrm{~g}$ & $10.00 \mathrm{a}$ & $0.000 \mathrm{a}$ & $12.33 \mathrm{a}$ & $5.333 \mathrm{ab}$ & $6.333 \mathrm{a}$ & $9.333 \mathrm{a}$ & $4.000 \mathrm{a}$ & $4.667 \mathrm{a}$ & $5.000 \mathrm{a}$ \\
\hline $\begin{array}{c}\mathrm{S}_{7}= \\
\text { Choygoria }\end{array}$ & $71.67 \mathrm{ef}$ & $6.000 \mathrm{bcd}$ & $0.000 \mathrm{a}$ & $\begin{array}{l}9.333 \\
\text { abcd }\end{array}$ & $\begin{array}{l}4.000 \\
\text { abcd }\end{array}$ & $3.000 \mathrm{bc}$ & $2.333 \mathrm{bc}$ & $0.000 \mathrm{c}$ & $3.667 \mathrm{ab}$ & $2.000 \mathrm{bcd}$ \\
\hline $\begin{array}{c}\mathrm{S}_{8}= \\
\text { Mirzanagar }\end{array}$ & $81.33 \mathrm{a}$ & $7.333 \mathrm{~b}$ & $0.000 \mathrm{a}$ & $5.667 \mathrm{de}$ & $\begin{array}{c}3.000 \\
\text { bcd }\end{array}$ & $3.333 \mathrm{bc}$ & $3.000 \mathrm{bc}$ & $2.333 \mathrm{~b}$ & $2.667 \mathrm{abc}$ & $2.000 \mathrm{bcd}$ \\
\hline $\begin{array}{c}\mathrm{S}_{9}= \\
\text { Nijkalikapur }\end{array}$ & $72.33 \mathrm{de}$ & $5.333 \mathrm{bcd}$ & $0.000 \mathrm{a}$ & $5.000 \mathrm{e}$ & $5.000 \mathrm{abc}$ & $3.333 \mathrm{bc}$ & $2.333 \mathrm{bc}$ & $0.333 \mathrm{c}$ & $2.333 \mathrm{bc}$ & $1.333 \mathrm{~d}$ \\
\hline $\mathrm{S}_{10}=$ Belonia & $77.00 \mathrm{bc}$ & $4.333 \mathrm{~cd}$ & $0.000 \mathrm{a}$ & $\begin{array}{l}7.667 \\
\text { bcde }\end{array}$ & $6.000 \mathrm{a}$ & $3.333 \mathrm{bc}$ & $4.667 \mathrm{~b}$ & $1.000 \mathrm{c}$ & $1.333 \mathrm{c}$ & $2.667 \mathrm{bcd}$ \\
\hline $\begin{array}{c}\text { s11 }= \\
\text { Parshuram }\end{array}$ & 69.33 ef & $4.333 \mathrm{~cd}$ & $0.000 \mathrm{a}$ & $7.000 \mathrm{cde}$ & $2.667 \mathrm{~cd}$ & $1.667 \mathrm{c}$ & $2.000 \mathrm{c}$ & $0.667 \mathrm{c}$ & $3.000 \mathrm{abc}$ & $2.667 \mathrm{bcd}$ \\
\hline $\begin{array}{c}\mathrm{S}_{12}=\text { Dhonik } \\
\text { unda }\end{array}$ & $71.67 \mathrm{ef}$ & $5.333 \mathrm{bcd}$ & $0.000 \mathrm{a}$ & $\begin{array}{l}8.333 \\
\text { abcde }\end{array}$ & $2.000 \mathrm{~d}$ & $2.333 \mathrm{bc}$ & $2.667 \mathrm{bc}$ & $0.000 \mathrm{c}$ & $2.333 \mathrm{bc}$ & $2.333 \mathrm{bcd}$ \\
\hline
\end{tabular}

Data presents the mean of three replications

Three hundred seeds were tested for each sample

Figure with common letters did not differ significantly at 5\% level by DMRT 
Table.4. Effect of variety on percent seed borne infection of rice seeds sample (based on lowest germination and highest prevalence of fungi)

\begin{tabular}{|c|c|c|c|c|c|c|c|c|c|c|}
\hline Variety & $\begin{array}{l}\text { Alternaria } \\
\text { padwickii }\end{array}$ & $\begin{array}{c}\text { Aspergill } \\
\text { us } \\
\text { niger }\end{array}$ & $\begin{array}{c}\text { Bipolaris } \\
\text { oryzae }\end{array}$ & $\begin{array}{c}\text { Curvularia } \\
\text { lunata }\end{array}$ & $\begin{array}{l}\text { Fusarium } \\
\text { monilifor } \\
\text { me }\end{array}$ & $\begin{array}{l}\text { Fusarium } \\
\text { oxysporum }\end{array}$ & $\begin{array}{c}\text { Nigrospora } \\
\text { oryzae }\end{array}$ & $\begin{array}{l}\text { Aspergillus } \\
\text { flavus }\end{array}$ & $\begin{array}{c}\text { Penicilliu } \\
m s p .\end{array}$ & Total \\
\hline $\begin{array}{l}\text { Joya } \\
\left(\mathrm{S}_{6}\right)\end{array}$ & $10.00 \mathrm{~b}$ & $0.000 \mathrm{~b}$ & $12.33 \mathrm{a}$ & $5.333 \mathrm{~b}$ & $6.333 \mathrm{a}$ & $9.333 \mathrm{a}$ & $4.000 \mathrm{a}$ & $4.667 \mathrm{~b}$ & $5.000 \mathrm{a}$ & 56.996 \\
\hline $\begin{array}{l}\text { BR6 } \\
\left(\mathrm{S}_{3}\right)\end{array}$ & $12.67 \mathrm{a}$ & $2.667 \mathrm{a}$ & $13.00 \mathrm{a}$ & $11.00 \mathrm{a}$ & $6.333 \mathrm{a}$ & $7.000 \mathrm{ab}$ & $3.667 \mathrm{a}$ & $5.333 \mathrm{~b}$ & $4.667 \mathrm{a}$ & 66.334 \\
\hline $\begin{array}{l}\text { Pajam } \\
\left(\mathrm{S}_{8}\right)\end{array}$ & $6.667 \mathrm{c}$ & $3.000 \mathrm{a}$ & $11.333 \mathrm{a}$ & $4.667 \mathrm{~b}$ & $4.667 \mathrm{a}$ & $6.333 \mathrm{~b}$ & $4.000 \mathrm{a}$ & $8.667 \mathrm{a}$ & $4.667 \mathrm{a}$ & 54.001 \\
\hline
\end{tabular}

\section{REFERENCES}

[1] Huda, M. Z. 2001. Regional development of irrigation technologies and its impact on food grain production in Bangladesh. M.S. Thesis, Department of Agricultural Economics, BAU Mymensingh, Bangladesh.

[2] BBS. 2008. Statistical Year Book of Bangladesh, Bangladesh Bureau of Statistics, Statistics Division, Ministry of planning, Government of People's Republic of Bangladesh, Dhaka, 2008.

[3] FAO. 2007. Production year Book. Food and Agriculture organization. Rome, Italy.

[4] BBS. 2007. Handbook of Agricultural Statistics. Bangladesh Bureau of Statistics, Statistics Division,Ministry of planning, Government of the people's republic of Bangladesh.

[5] Fakir, G. A. 1983. Teaching, Research and Training Activities on Seed Pathology in Bangladesh. Seed Sci. \& Tech. 11: 1345-1352.

[6] Ou, S. H. 1972. Rice diseases. Commonwealth Mycological Institute, Kew, Surrey. England.p. 368.

[7] ISTA. 1976. International Rules for Seed Testing Association. Int. Seed test. Assoc. 31: 107-115.

[8] Ramnath, S. B. Mathur and P. Neergaard. 1970. Seed-borne fungi of mung bean (Phaseolus aureus Roxb.) from India and their significance. Proc. Int. Seed Test. Asso. 35: 225-241.

[9] Kulshrestha, D. D., S. B. Mathur and P. Neergaard. 1976. Identification of seed-borne species of Colletotrichum freesia. 11: 116-125.

[10] Malone, G. P. and A. E. Muskette. 1964. Seed-borne fungi Description of 77 fungal species. Proc. Int. Seed Test. Asso. 29(2): 180-183.

[11] Booth, C. 1971. The genus Fusarium Commonwealth Mycol. Inst. Kew, Surrey, England. p.236.

[12] Ellis, M. B. 1971. Dematiaccous Hypomycetes. CMI, Kew, Surrey, England. p.507.

[13] Fakir, G. A., I. Hossain, M. U. Ahmad, M. Asad-ud-Doullah and M. Alam. 2002. Quality of farmer's Boro and T. Aman rice seeds collected before sowing from Bogra, Rajshahi and Rangpur Districts of Bangladesh. A paper presented in the Review and planning meeting of the rice seed health improvement (PETRRA) project, held on 21-22 April at BRRI, Gazipur, Bangladesh.

[14] Sharma, O. P., A. Vaid, S. C. Safi, J. Saxena and R. C. Dubey. 1997. Mycoflora of discoloured rice grains and its pathogenic potential in Himachal Pradesh. Himalayan Micro. Diver. Part-1: 273-281.

[15] Rahman, M. M. 2002a. Effect of farmers storing processes in relation to seed quality and seed-borne fungi of rice (BR11). M.S. Thesis, Dept. of Plant Pathology, BAU, Mymensingh.p.52. 